\title{
Study of depressed mood and quality of life in asthma patients in Tehran using the 28-item general health questionnaire
}

\author{
S.F. Tafti, ${ }^{1}$ A. Cheraghvandi, ${ }^{1}$ M. Safa, ${ }^{1}$ D.F. Eragh, ${ }^{7}$ B. Mokri ${ }^{1}$ and F. Talischi ${ }^{7}$
}

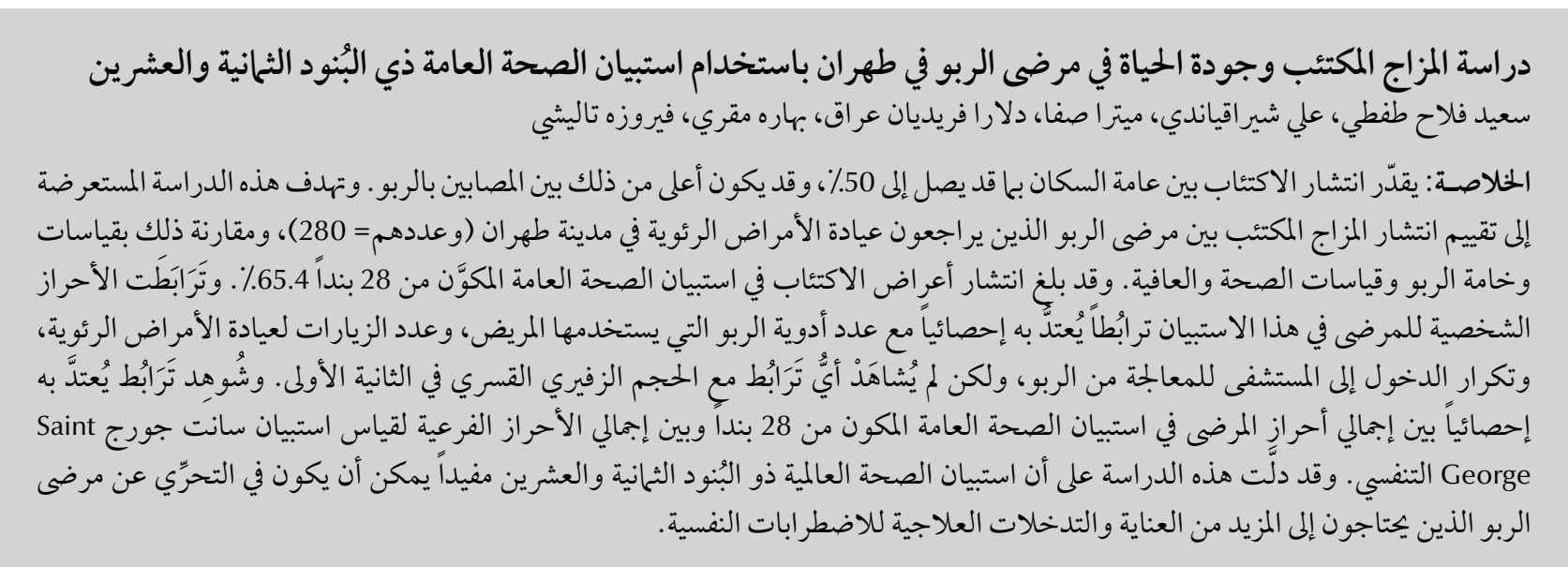

ABSTRACT The prevalence of depression among the general population has been estimated as up to $50 \%$ and even higher among asthmatics. The aim of this cross-sectional study was to evaluate the prevalence of depressed mood among asthma patients $(n=280)$ attending a pulmonary clinic in Tehran and compare it with measures of severity of asthma and of health and well-being. The prevalence of depression symptoms on the 28-item general health questionnaire (GHQ-28) was 65.4\%. Patients' individual scores on the GHQ-28 were significantly correlated with the number of asthma medications used, frequency of visits to the pulmonary clinic and frequency of hospitalizations for asthma, but not with FEV1. A significant correlation was found between patients' total scores on the GHQ-28 and total and subscale scores on the Saint George respiratory questionnaire. The GHQ-28 may be useful for screening for asthma patients who need more attention and therapeutic intervention for psychiatric disorders.

Étude de la dépression et de la qualité de vie chez des patients asthmatiques à Téhéran à l'aide du questionnaire général sur la santé en 28 items

RÉSUMÉ Selon les estimations, la prévalence de la dépression peut atteindre $50 \%$ dans la population générale et elle est même supérieure chez les asthmatiques. L'objectif de la présente étude transversale était d'évaluer la prévalence de la dépression chez des patients asthmatiques $(n=280)$ consultant dans un centre de soins des maladies pulmonaires à Téhéran et de la comparer aux mesures de sévérité de l'asthme, de l'état de santé général et de bien-être. La prévalence des symptômes de dépression dans le questionnaire général sur la santé en 28 items (GHQ-28) était de 65,4\%. Les scores individuels des patients au questionnaire étaient fortement corrélés au nombre de médicaments utilisés pour traiter l'asthme, à la fréquence des visites au centre de soins des maladies pulmonaires et à la fréquence des hospitalisations induites par l'asthme, mais ils n'avaient pas de lien avec le résultat du volume expiratoire maximum par seconde (VEMS). Une corrélation importante a été retrouvée entre le score total des patients au questionnaire GHQ-28 et le score total au Questionnaire respiratoire de St-George mais aussi les résultats pour les sous-échelles de ce dernier. Le questionnaire GHQ-28 peut être utile pour dépister les patients asthmatiques nécessitant une attention accrue et une intervention thérapeutique visant les troubles psychiatriques.

'Massih Daneshvari Hospital, National Research Institute of Tuberculosis and Lung Diseases, Shahid Beheshti University of Medical Sciences, Tehran, Islamic Republic of Iran (Correspondence to F. Talischi:firouzehtalischi@yahoo.com).

Received: 01/06/09; accepted: 11/04/10 


\section{Introduction}

The prevalence of depression has been estimated to be up to $50 \%$ among the general medical population and even higher among asthmatics [1-4]. Children with moderate to severe asthma have more psychiatric and general health ailments and social difficulties than those with mild asthma [4]. Furthermore the severity of asthma seems to be influenced by conditions such as psychiatric disorders and anxiety. These symptoms can be confused with those of asthma and lead to unnecessary use of asthma medication [3]. Emotions such as anxiety, anger, happiness, excitement, satisfaction and neutral emotions have been shown to influence forced expiratory volume in 1 second (FEV1), peak expiratory flow rate and airways resistance in asthma patients [5].

Diagnosis of psychiatric conditions and mood disorders such as depression symptoms may not be possible in a general medical visit. Yet it is not practical for all patients to be evaluated by a psychiatrist, and therefore instruments such as the general health questionnaire (GHQ), which measures psychological distress related to general medical illness, are useful for screening patients in the physician's office [6]. The 28-item version of the questionnaire (GHQ-28) has been developed using factor analysis and its main application has been for research purposes. Scores on the GHQ-28 subsections can be used for screening for anxiety and depression [7]. The questionnaire has previously been translated into Farsi and its validity and reliability accepted in other studies in the Islamic Republic of Iran $[8,9]$.

The purpose of this study in Tehran was to evaluate the prevalence of depression symptoms among asthma patients using the Farsi version of the GHQ-28 and compare it with measures of the severity of asthma and health and well-being.

\section{Methods}

\section{Study design and sample}

This was cross-sectional study of 280 asthmatic patients referred to the spirometry laboratory from the Massih Daneshvari hospital pulmonary clinic between July and September 2007. The hospital is a quaternary referral centre and serves a diverse population of asthmatics of different social and economic status [10]. The spirometry laboratory receives referrals from different parts of Tehran and elsewhere in the Islamic Republic of Iran.

Patients were included if they agreed to participate in the study, had a previous diagnosis of asthma and were in a stable medical condition with no other serious underlying medical and disabling conditions. Patients who needed hospitalization or were oxygen dependent and had prescriptions to continue using oxygen at home were excluded from the study.

\section{Data collection}

Demographic information was collected from patients, including age, sex, marital status, literacy and household size (number of people living in the same household).

All patients completed the Farsi version of the GHQ-28 and a patient information form. The translation to Farsi and assessment of the validity and reliability of the GHQ-28 has been reported before [9]. Total score range from 0 to 84 in 4 subscales measuring somatic symptoms; anxiety/insomnia; social dysfunction; and severe depression. The cut-off score for depression symptoms was $>22$.

Patients also completed the Farsi version of the Saint George respiratory questionnaire (SGRQ). The SGRQ measures impaired health and perceived quality of life in patients with airways disease. It has been standardized for chronic obstructive pulmonary patients and has been previously translated and validated in Farsi [11]. Similar to the original English version, the Farsi version has 50 questions with 3 subscales covering: symptoms (frequency and severity of respiratory symptoms); activities (activities that cause or are limited by breathlessness); and impact (social functioning, psychological disturbances resulting from airways disease). It is scored from 0 (optimal health) to 100 (worst health) with total score $<10$ considered normal [9].

Other measures of disease activity were recoded on a separate form, including FEV1, number of asthma control medications used, frequency of visits to the pulmonary clinic in the past year and frequency of hospital admissions for asthma exacerbations in the past year [12].

\section{Analysis}

Data was entered and analysed using SPSS, version 13, statistical software. The GHQ-28 questionnaire results were compared with the other measures of disease activity (SGRQ scores, FEV1 values, number of asthma control medications, frequency of clinic visits and hospital admissions related to asthma). The correlations between variables were evaluated using the chi-squared test and t-test.

\section{Results}

\section{Background characteristics}

The study participants were 280 asthmatics, with a mean age of 43.8 [standard deviation (SD) 16.6] years, range 16-96 years. There were 151 women (53.9\%) and 129 men (46.1\%). A majority of the patients $(72.1 \%)$ were married and had a literacy level of education (81.8\%). The mean household size was 4.2 (SD 1.8), range 1-12. Almost all respondents had a household heating system (except those living in warm climate). Only $3.2 \%$ were smokers.

Based on Jones' criteria, $15.0 \%$ of the patients had mild intermittent asthma, 
$33.9 \%$ mild persistent asthma, $27.9 \%$ moderate persistent asthma and $21.1 \%$ severe persistent asthma; data were unavailable for $2.1 \%(n=6)$ patients. According to patients' self-reports or the medical records $50.0 \%$ of patients had other medical conditions. The mean number of asthma medications taken were 2.4 (SD 1.9), range 0-8; mean number of pulmonary clinic visits in the past year was 9.2 (SD 17.0), range 0-200; and mean number of hospitalizations for asthma in the past year was 0.5 (SD 1.7), range 0-20. The mean predicted FEV1 of this group of patients was $74.6 \%$ (SD 22.9\%). The total mean score on the SGRQ was 50 (SD 20) and mean scores on the subscales were 65 (SD 20) for symptoms, 50 (SD 22) for activity and 44 (SD 24) for impact.

\section{Prevalence of depression symptoms}

Of the 280 asthma patients $183 \mathrm{had}$ depression symptoms based on the GHQ-28 cut-off score of 22, giving an overall prevalence of depressed mood of $65.4 \%$. Significantly more women had depression symptoms than did men $(70.2 \%$ versus $54.9 \%)(P=0.005)$. Rates of depressed mood was also higher among patients who were illiterate compared with those who were educated $(77.4 \%$ versus $59.3 \%)$ ( $P$ $=0.009)$ and among those who had comorbid illness compared with those who did not $(71.5 \%$ versus $54.6 \%)(P=$ 0.002) (Table 1).

Patients' individual scores on the GHQ-28 were not significantly correlated with their FEV1 values (Table 2). However, other measures of asthma severity - number of asthma medications used, frequency of visits to the pulmonary clinic for asthma and frequency of hospitalizations for asthma exacerbations - showed a good correlation with the GHQ-28 total score (all $P<0.001)$ (Table 2).

A significant correlation was found between patients' individual total score on the GHQ-28 and score on the

\begin{tabular}{|c|c|c|c|}
\hline \multirow[t]{2}{*}{ Variable } & \multicolumn{2}{|c|}{ Patients with depression ${ }^{\mathrm{a}}$} & \multirow[t]{2}{*}{$P$-value ${ }^{b}$} \\
\hline & No. & $\%$ & \\
\hline Sex & & & 0.005 \\
\hline Male & 90 & 54.9 & \\
\hline Female & 118 & 70.2 & \\
\hline Marital status & & & 0.810 \\
\hline Married & 148 & 62.2 & \\
\hline Single & 56 & 63.6 & \\
\hline Literacy & & & 0.009 \\
\hline Illiterate & 48 & 77.4 & \\
\hline Literate & 160 & 59.3 & \\
\hline Comorbid illness & & & 0.002 \\
\hline Yes & 113 & 71.5 & \\
\hline No & 95 & 54.6 & \\
\hline Smoker & & & 0.342 \\
\hline No & 10 & 50.0 & \\
\hline Yes & 192 & 62.7 & \\
\hline
\end{tabular}

${ }^{a} G H Q-28$ cut-off $>22$. bPearson $\chi^{2}$.
SGRQ (both total score and scores on subscales) $($ all $P<0.001)($ Table 3$)$.

\section{Discussion}

Depression symptoms are relatively common among asthma patients $[1,2]$, and emotions such as anxiety, anger, happiness, excitement, satisfaction and neutral emotions can influence respiratory parameters [5]. In our group of patients who were referred to the spirometry laboratory from the pulmonary clinic, the prevalence of depression according to the GHQ-28 was $65.4 \%$.

The GHQ questionnaire is known as a practical tool for assessing depression symptoms [1]. Additionally, it has been translated to Farsi and its validity and reliability evaluated and found to be acceptable in other studies [8,9]. Islamic Republic of Iran, 204 individuals 50 years and older completed the GHQ-28 questionnaire and were also evaluated for depression by a psychiatrist. The Cronbach alpha coefficient In a study by Malakouti et al. in the

\begin{tabular}{lcc}
\hline $\begin{array}{l}\text { Table } 2 \text { Correlation between asthma patients' } \\
\text { health questionnaire (GHQ-28) }\end{array}$ and their illness characteristics $(\boldsymbol{n}=\mathbf{2 8 0})$ \\
\hline Variable & $\begin{array}{c}\text { Correlation with total GHQ-28 } \\
\text { Kendall's tau }\end{array}$ & $\begin{array}{l}\boldsymbol{P} \text {-value } \\
\text { No. of inhalants used }\end{array}$ \\
No. of clinic visits in the past year & 0.136 & 0.001 \\
No. of hospitalizations in the past year & 0.216 & 0.001 \\
Visual analogue scale & 0.193 & 0.001 \\
Katz index score & 0.241 & 0.001 \\
FEV1 & 0.412 & 0.001 \\
\hline
\end{tabular}

$F E V I=$ forced expiratory volume in 1 second. 


\begin{tabular}{|c|c|c|}
\hline \multirow[t]{2}{*}{ SGRQ subscale } & \multicolumn{2}{|c|}{ Correlation with total GHQ-28 } \\
\hline & Kendall's tau & $P$-value \\
\hline Symptoms & 0.281 & 0.001 \\
\hline Activity & 0.299 & 0.001 \\
\hline Impact & 0.420 & 0.001 \\
\hline Total & 0.412 & 0.001 \\
\hline
\end{tabular}

for the questionnaire was 0.89 and repeatability coefficient was 0.58 , which were appropriate and acceptable. Using the area under the receiver operating characteristics curve, a cut-off point of 19/20 was used for psychiatric illness and was compared with the psychiatrist's diagnosis with sensitivity of 0.83 and specificity of 0.78 [9]. Studies have also shown that the severity of psychiatric symptoms is related to the score [8]. The questionnaire is sensitive for mood disorders [13].

In this study, no significant correlation was found between the scores on the GHQ-28 and FEV1 in our sample of asthma patients. However, a good correlation was found between scores on the GHQ-28 and the SGRQ. There was also a significant correlation between depressed mood and the number of asthma medications, frequency of clinic visits for asthma and number of hospitalizations for asthma exacerbations. It can be concluded that asthma may be emotionally debilitating and even influence mood disorders such as depression.

Gender and social status are known to influence the GHQ results [13]. This was supported in our study where demographic factors such as illiteracy, which has been related to poorer social status, were significantly associated with GHQ-28 scores.

Some patients are considered to have "difficult-to-control" asthma and relative insensitivity to glucocorticoid treatment, which has been attributed mostly to poor compliance with treatment and genetic factors as well as psychological and psychiatric disorders [14]. Emotional stress is also considered an exacerbating factor for asthma. Emotional expressions such as laughing, crying, anger or fear and panic attack can induce hyperventilation and hypocapnia and airways narrowing. In cases of difficult-to-control asthma psychological and psychiatric disorders need to be considered, after the correct diagnosis and compliance with treatment have been confirmed and other factors such as smoking and comorbidities have been investigated. Psychiatric disorders can be considered as diseases alongside sinusitis, gastroesophageal reflux disease, obesity/obstructive sleep apnoea and need to be addressed and appropriately treated [14].

There were some limitations to this study. The patients included were outpatients referred from the pulmonary clinic to the spirometry laboratory. Hospitalized asthmatics may have an even higher incidence of depression symptoms. This study was cross-sectional with no control group of general patients to compare the incidence of depression symptoms. We plan to follow the study with a validation study by formal psychiatric evaluation of the patients. Also, the GHQ questionnaire content does not discriminate between psychological distress and respondents' personal beliefs and feelings.

Considering the high incidence of depression symptoms in the study population of asthmatics, the GHQ-28 may be useful to screen for patients who need more attention and therapeutic intervention for psychiatric disorders.

\section{Acknowledgements}

Researchers would like to thank colleagues at Massih Daneshvari Hospital Research Centre for assistance in performing this study.

\section{References}

1. Zielinski T et al. Depression in asthma: prevalence and clinical implications. Primary Care Companion to the Journal of Clinical Psychiatry, 2000, 2:153-158.

2. Kaplan HI, Sadock BJ. Synopsis of psychiatry: behavioral sciences/clinical psychiatry, 8th ed. Philadelphia, Lippincott Williams and Wilkins, 1998.

3. Anbar RD. Self-hypnosis for anxiety associated with severe asthma: a case report. BMC Pediatrics, 2003, 3:7.

4. Varni JW, Limbers CA, Burwinkle TM. Impaired health-related quality of life in children and adolescents with chronic conditions: a comparative analysis of 10 disease clusters and 33 disease categories/severities utilizing the Peds QL TM 4.0 Generic Core Scales. Health and Quality of Life Outcomes, 2007, 5:43.
5. Ritz T, Steptoe A. Emotion and pulmonary function in asthma; reactivity in the field and relationship with laboratory induction of emotion. Psychosomatic Medicine, 2000, 62:808-815.

6. Goldberg DP, Blackwell B. Psychiatric illness in general practice: a detailed study using new method of case identification. British Medical Journal, 1970, 2:439-443.

7. Nagyova I et al. General Health Questionnaire-28: psychometric evaluation of the Slovak version. Studia Psychologica, 2000, 42:351-361.

8. Abdi Masoul F et al. [Evaluation of relation between job stress and psychiatric well being: study of 200 nurses]. Journal of Tehran University of Medical Sciences, 2007, 65(6):65-75 [In Farsi]. 
9. Malakouti SK et al. Reliability, validity and factor structure of the GHQ-28 used among elderly Iranians. International Psychogeriatrics, 2007, 19:623-634.

10. Li L, Wang HM, Shen Y. Chinese SF-36 health survey: translation, cultural adaptation, validation, and normalization. Journal of Epidemiology and Community Health, 2003, 57:259-263.

11. Tafti SF et al. [Evaluation of reliability and validity of the Farsi version of the Saint George Respiratory Questionnaire for patient with chronic respiratory pulmonary disease (COPD)]. Pajhouhandeh Journal, 1384, 55(1) [In Farsi].
12. Incalzi RA et al. Evaluation of health outcomes in elderly patients with asthma and COPD Using disease-specific and generic instruments. Chest, 2001, 120:734-742.

13. Rush JA. Handbook of psychiatric measures. Arlington, Virginia, American Psychiatric Association, 2008:70.

14. Smith JR et al. Psycho-educational interventions for adults with severe or difficult asthma: a systemic review. Journal of Asthma, 2007, 44(3):219-241.

\section{Global status report on noncommunicable diseases 2010}

The Global status report on noncommunicable diseases 2010 is the first report on the worldwide epidemic of cardiovascular diseases, cancer, diabetes and chronic respiratory diseases, along with their risk factors and determinants.

Noncommunicable diseases killed 36 million people in 2008, and a large proportion of these deaths occurred before the age of 60 years, so during the most productive period of life. The magnitude of these diseases continues to rise, especially in low- and middle-income countries.

This report reviews the current status of noncommunicable diseases and provides a roadmap for reversing the epidemic by strengthening national and global monitoring and surveillance, scaling up the implementation of evidence-based measures to reduce risk factors like tobacco use, unhealthy diet, physical inactivity and harmful alcohol use, and improving access to cost-effective healthcare interventions to prevent complications, disabilities and premature death. This report also provide a baseline for future monitoring of trends and for assessing the progress Member States are making to address the epidemic.

The report was developed as part of the implementation of the 2008-2013 Action Plan for the Global Strategy for the Prevention and Control of Noncommunicable Diseases, which was endorsed by the World Health Assembly in 2008.

Further information about this and other WHO publications is available at: http:/ /www.who.int/publications/en/ 